\title{
Human Cases of Fascioliasis in Fujian Province, China
}

\author{
Lin Ai, Yu-Chun Cai, Yan Lu, Jia-Xu Chen, Shao-Hong Chen* \\ National Institute of Parasitic Diseases, Chinese Center for Disease Control and Prevention, WHO Collaborating Center for Tropical Diseases, Key \\ Laboratory of Parasite and Vector Biology, National Health and Family Planning Commission, Shanghai 200025, China
}

\begin{abstract}
Fascioliasis is a foodborne zoonotic parasitic disease. We report 4 cases occurring in the same family, in whom diagnosis of acute fascioliasis was established after series of tests. One case was hospitalized with fever, eosinophilia, and hepatic lesions. MRI showed hypodense changes in both liver lobes. The remaining 3 cases presented with the symptom of stomachache only. Stool analysis was positive for Fasciola eggs in 2 adult patients. The immunological test and molecular identification of eggs were confirmed at the National Institute of Parasitic Diseases, Chinese Center for Disease Control and Prevention, Shanghai, China. The results of serological detection were positive in all the 4 patients. DNA sequencing of PCR products of the eggs demonstrated $100 \%$ homology with ITS and cox 1 of Fasciola hepatica. The conditions of the patients were not improved by broad-spectrum anti-parasitic drugs until administration of triclabendazole.
\end{abstract}

Key words: Fasciola hepatica, human case, diagnosis, triclabendazole

\section{INTRODUCTION}

Fascioliasis, one of the major veterinary parasitic diseases in ruminants, such as cattle, sheep, and goats, causes economic losses in animal husbandry [1,2]. Human fascioliasis has been ignored for a long time until the last decade, when this zoonotic infection emerged or reemerged in more than 60 countries $[3,4]$. Humans are accidental hosts by ingesting raw fresh water plants or drinking water contaminated with metacercariae [5]. Recently, it was conservatively estimated that least 2.65 million individuals suffer from this disease worldwide [6]. According to such emergence, pathogenicity, and immunological interactions [7-10], human fascioliasis was labeled as a neglected tropical diseases (NTD) by the World Health Organization (WHO) [11], with chronic, debilitating, and poverty-promoting characters [1-3,12].

In China, fascioliasis is also neglected and usually misdiagnosed as a liver cancer. Attention was not paid to disease prevalence, diagnosis, and treatment in China. Few published articles are available on human fascioliasis, with only scattered cases reported long time ago [13-16]. The first outbreak of hu-

- Received 12 November 2016, revised 3 January 2017, accepted 15 January 2017. *Corresponding author (chensh637@163.com)

(c) 2017, Korean Society for Parasitology and Tropical Medicine

This is an Open Access article distributed under the terms of the Creative Commons Attribution Non-Commercial License (http://creativecommons.org/licenses/by-nc/4.0) which permits unrestricted non-commercial use, distribution, and reproduction in any

medium, provided the original work is properly cited. man fascioliasis in China was in Binchuan (southwest China), in January 2012. After morphological, immunological, and molecular characterizations, F. gigantica was considered as a pathogen attracting attention from the Chinese government in finding ways to prevent and control this ailment [17]. Another important human fascioliasis case was a young man, 24 years old. After routine blood analysis, as well as pathological, immunological, ultrasonic, and molecular examinations, definite diagnosis of F. hepatica infection was reached for this case [18].

This study aimed to report clinical, parasitological, immunological, and molecular features of 4 patients in a single family suffering from fascioliasis in Fujian, China.

\section{CASE RECORD}

Four patients, including Mrs. Xiao, her husband, daughter, and younger sister, were residents of Changle County, Fujian province, and consumed water dropwort cultured around their residence in December 2015. All of them were in good health until April 2016, when they developed stomachache. Mrs. Xiao's symptoms were most serious among the 4 family members, with overt marasmus without fever. From May to June 2016, she presented episodic right upper quadrant (RUQ) abdominal pain which spread to the right shoulder with finger as well as chin aches. Beside stomachache, she also developed severe diarrhea ( $>20$ times/per day), occasional bloating, fever $\left(39-40^{\circ} \mathrm{C}\right)$, chills, occasional dizziness, weakness, fatigue, in- 
creased blood eosinophil and granulocyte counts, jaundice, body hives, and ostealgia, and hated the cold weather and light. Moderate symptoms were found in her husband, daughter, and younger sister, who only showed abdominal pain without lasting high fever.

In June 2016, detailed clinical, laboratory, and radiographic tests were performed in Fuzhou General Hospital of Nanjing Military Region, Nanjing, China. Laboratory tests revealed high eosinophilia, mild normocytic normochromic anemia, and high serum activity of alkaline phosphatase (ALP), with normal alanine aminotransferase (ALT) and aspartate amino transferase (AST) levels. MRI showed hypodense changes (total

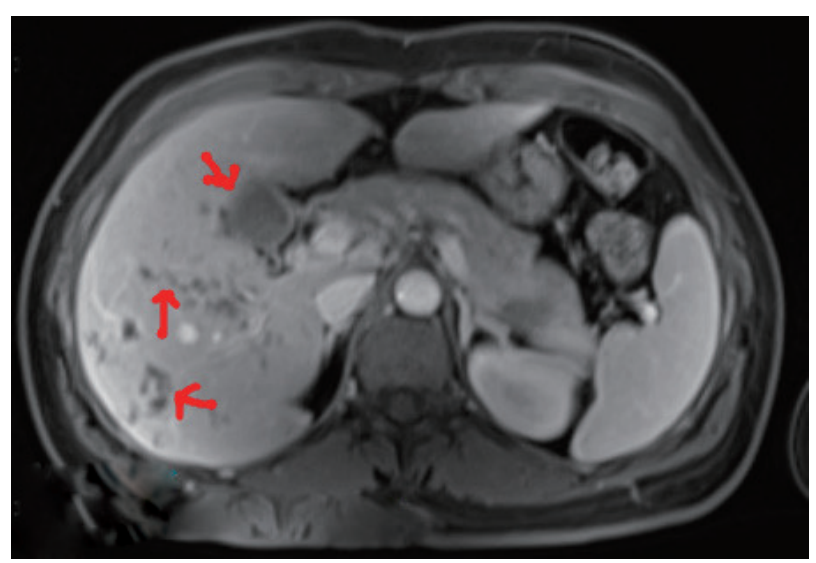

Fig. 1. Abdominal magnetic resonance imaging (MRI) showing multiple hypodense foci with subcapsular location.

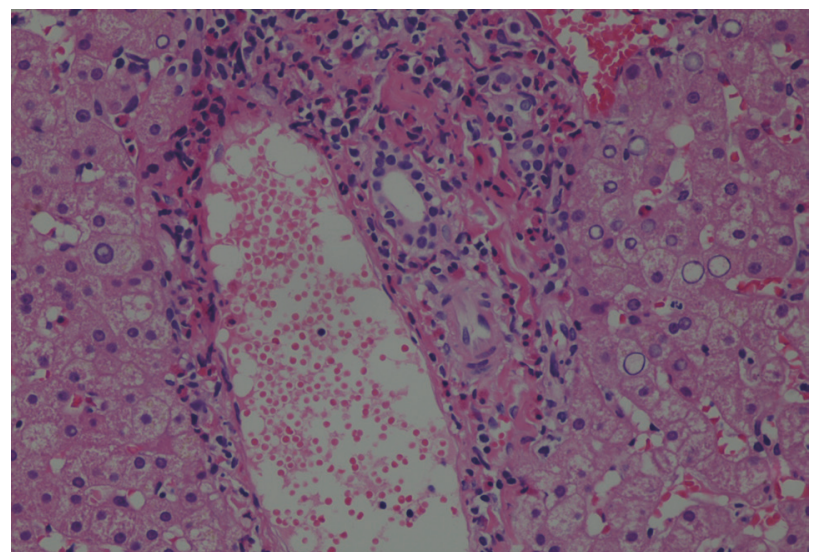

Fig. 2. Pathological section: a small portion of the obtained material comprised hepatocytes, while the rest was made of young granulation tissue infiltrated by eosinophils and other inflammatory cells. Portal fields were normal in size, mild to moderately infiltrated, mainly by eosinophils, with small amounts of other inflammatory cells. Glycogenated nuclei were observed in the parenchyma. Inflammatory cells of the same types found in portal fields were observed in the sinusoids (HE stain, $\times 200$ ). diameter up to $6 \mathrm{~cm}$ ) in both liver lobes below the diaphragm, predominantly localized posteriorly and centrally (Fig. 1).

Because malignancy was initially considered, laparoscopic liver biopsy under visual control was performed at the Department of surgery on 23 June 2016. The liver biopsy was then obtained which showed liver tissue with eosinophil accumulation around the hepatobiliary system. Moreover, a detailed reevaluation of liver biopsy in National Institute of Parasitic Diseases, Chinese Center for Disease Control and Prevention (NIPD) (15 August 2016) revealed histopathological features suggesting a parasitic infection (Fig. 2).

Upon admission to Fuzhou General Hospital of Nanjing Military Region, Mrs. Xiao's temperature was $39-40^{\circ} \mathrm{C}$. Abdominal examination showed localized tenderness over RUQ and a palpable liver edge below the right costal margin. The rest of the physical examination was unremarkable. On admission, the following abnormal blood parameters were shown: albumin levels, 37.3 (decreased; the normal value is $65-85 \mathrm{~g} /$ L); erythrocyte count, $3.84 \times 10^{12} / \mathrm{L}$ (decreased, the normal value is 4.0-5.1 $\left.\times 10^{12} / \mathrm{L}\right)$; eosinophilic granulocyte count, $2.9 \times 10^{9} /$ $\mathrm{L}$ (sharply increased; the normal value is $0.02-0.52 \times 10^{9} / \mathrm{L}$ ); the percentage of eosinophilic granulocyte was $33.5 \%$ (sharply increased; the normal value is $0.4-8 \%$ ); platelet count, $424 \times 10^{9}$ / $\mathrm{L}$ (increased; the normal value is $125-350 \times 10^{9} / \mathrm{L}$ ). Tumor markers (CEA, CA 19.9, and AFP) were within the normal range. Serum biochemical analysis revealed that the values of $\mathrm{Fe}$, ALT, and AST were normal. However, the value of ALP, alpha-amylase and direct bilirubin increased $(135 \mathrm{U} / \mathrm{L}, 185 \mathrm{U} / \mathrm{L}$ and $6.0 \mu \mathrm{M} / \mathrm{L}$; the normal value is $35-130 \mathrm{U} / \mathrm{L}, 72-182 \mathrm{U} / \mathrm{L}$ and $<5 \mu \mathrm{M} / \mathrm{L}$, respectively).

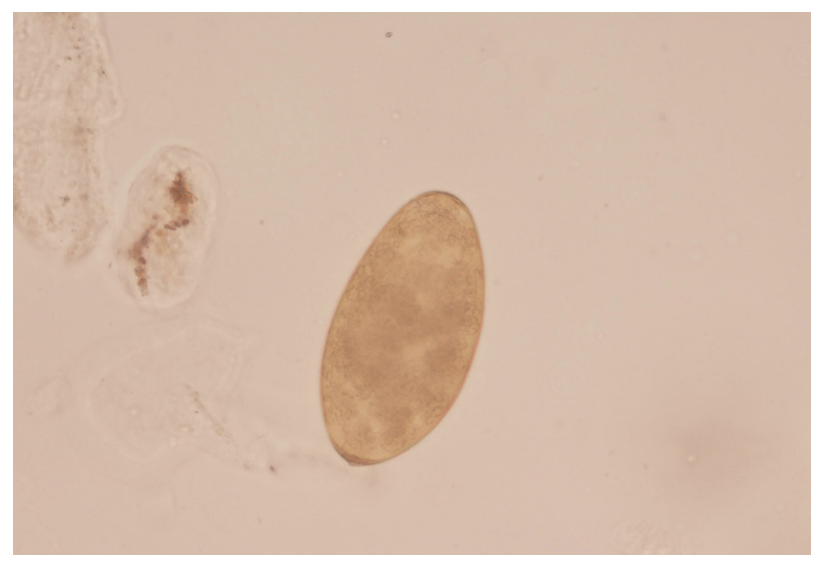

Fig. 3. Operculated egg of Fasciola hepatica $(143 \times 82 \mu \mathrm{m})$ evidenced in stool samples by microscopy $(\times 40)$. 
Based on routine, the doctors suspected a parasitic disease for the 4 patients, who were referred to Fujian Center for Control and Prevention. By stool sample analysis using water sedimentation method, Fasciola spp. eggs were found in Mrs. Xiao and her younger sister. Based on the shape and mean size $(143 \times 82 \mu \mathrm{m})$, the operculated eggs were identified as Fasciola spp. eggs (Fig. 3). Since the other 2 family members also consumed water dropwort and showed stomachache, all of them were referred to NIPD for further diagnosis.

Parasitic diagnostic tests, including morphological, immunological, and molecular analyses, were carried out in NIPD, China CDC. Repeated stool examinations were positive for Fasciola eggs in Mrs. Xiao and the younger sister. Serum analysis was completed with a DRG human Fasciola-specific IgG antibody detection kit (DRG-international Company, Marburg, Germany), which has already been evaluated in front of different epidemiological situations by WHO [19], with positive results obtained in all 4 patients. Moreover, because no Fasciola eggs and other parasite eggs were found in Ms. Xiao' daughter and husband, IgG antibodies to some common parasites, including Paragonimus westermani, Clonorchis sinensis, cysticercus, plerocercoid, Schistosoma japonicum, and Echinococcus, have also been detected by commercial ELISA kits (Shenzhen Combined Biotech Company, Shenzhen, China) with negative results.

Furthermore, Fasciola eggs from Ms. Xiao's and the younger sister's fecal samples were respectively assessed by molecular methods to identify the pathogenic species. Firstly, $5 \mathrm{~g}$ feces of Mrs. Xiao and her sister were respectively recovered using a standard washing-sieving procedure [20] and then resuspended in physiological saline to give a pool of eggs in a final volume of $20 \mathrm{ml}$. For the extraction of genomic DNA, 35 eggs from each patient were respectively isolated and pipetted into 2 eppendorf tube and covered with $30 \mu \mathrm{l}$ distilled water. Each sample of eggs was vortexed with glass beads for $30 \mathrm{~min}$, to disrupt the egg shells, before the DNA was extracted with SDS/ proteinase K [21], and the commercial QIAamp DNA extraction kit (Qiagen Inc., Valencia, California, USA) according to the manufacturer's instructions. DNA samples were stored at $-20^{\circ} \mathrm{C}$ until use. The DNA region comprising part of ITS sequences and a portion of the cox 1 gene ( $\mathrm{p} c o x 1$ ) were amplified by PCR. The primers and PCR reaction systems were according to the research from Luton et al. [22] and Bowles et al. [23], respectively. The pITS and pcox1 sequences are available from DDBJ, EMBL, and GenBank ${ }^{\mathrm{TM}}$ under the accession nos. shown in Table 1. The 2 ITS PCR products were subjected to direct sequencing with $945 \mathrm{bp}$ band obtained. The sequences included the part ITS-1 (422 bp), 5.8S (162 bp), and part ITS-2 (361 bp) sequences. Agarose gel electrophoresis results are shown in Fig. 4A. Agarose gel electrophoresis can be seen in Fig. 4B. GenBank ${ }^{\mathrm{rm}}$ accession nos. of pITS and pcox1 sequences of the 2 patients and reference sequences used for comparison were shown in Table 1. DNA sequencing of PCR products of the eggs demonstrated $100 \%$ homology with ITS and $\operatorname{cox} 1$ of $F$. hepatica.

After the diagnosis was confirmed (June 2016) in Fuzhou, triclabendazole (Egaten ${ }^{\circledR}$, Novartis, Basel, Switzerland) was administered at a dose of $10 \mathrm{mg} / \mathrm{kg}$ for per day to Mrs. Xiao, her daughter, and younger sister (her husband went out to business, and received medical treatment only in August). Only 1 dose was used in all 4 patients. After taking this medicine for 1 course ( $250 \mathrm{mg} /$ tablet, 8 tablets), the symptoms of Ms. Xiao's sister, daughter, and husband disappeared. After oral medication in the first and second days, Mrs. Xiao had nausea. A day later, the latter symptom was also alleviated. However, she had RUQ abdominal pain, high fever $\left(38^{\circ} \mathrm{C}\right)$, and urticaria. Before Ms. Xiao was discharged on 22 August 2016 (taking triclabendazole for 3 courses totally), the following parameters were obtained: leukocytes, $8.73 \times 10^{9} / \mathrm{L}$ with eosinophilia at $22.7 \%$; platelets, 328×10/L; AST, 23 U/L; ALT, 15.2 U/L; ALP, 100 U/

Table 1. Fasciola samples of the patients from Fujian, China used in the present study, as well as their GenBank ${ }^{\mathrm{TM}}$ accession nos. for sequences of part internal transcribed spacers (ITS) of nuclear ribosomal DNA (rDNA), a portion of mitochondrial DNA (mtDNA) cytochrome $c$ oxidase subunit 1 (pcox1) and other reference sequences

\begin{tabular}{|c|c|c|c|c|c|}
\hline \multirow{2}{*}{ Sample codes } & \multirow{2}{*}{ Location } & \multirow{2}{*}{ Stage } & \multirow{2}{*}{ Identification by morphology } & \multicolumn{2}{|c|}{ GenBank $^{\mathrm{TM}}$ accession no. } \\
\hline & & & & ITS & $\operatorname{cox} 1$ \\
\hline Fhfj1 & China & Egg & Fasciola hepatica & KX856339 & KX856337 \\
\hline Fhfj2 & China & Egg & F. hepatica & KX856340 & KX856338 \\
\hline Fh & Australia & Adult & F. hepatica & / & NC_002546 \\
\hline Fh & USA & Adult & F. hepatica & JF708031 & \\
\hline
\end{tabular}




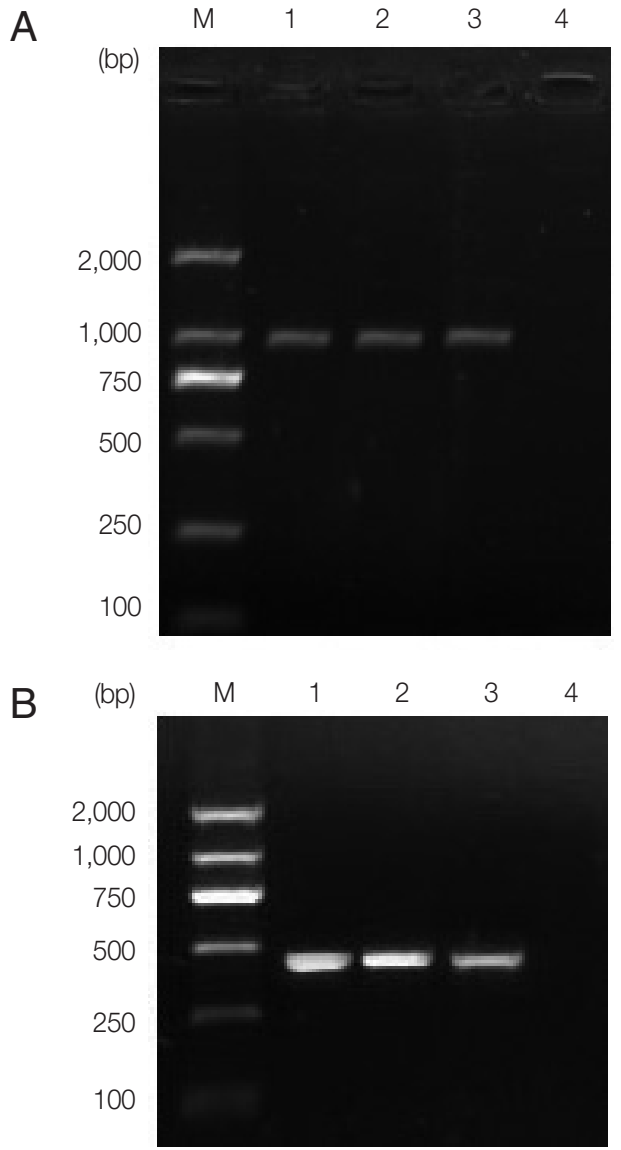

Fig. 4. (A) Agarose gel electrophoresis analyzing ITS PCR products of representative Fasciola hepatica from 2 patients. Lanes 1 , 2 , and 3, PCR results of patient samples and $F$. hepatica positive control, respectively; Lane 4, negative control; $M$, represents a DNA size marker (ordinate values in bp). (B) Agarose gel electrophoresis analyzing pcox1 PCR products of representative $F$. hepatica from 2 patients. Lanes 1, 2, and 3, PCR results of sample from patients and $F$. hepatica positive control, respectively; Lane 4, negative control; $\mathrm{M}$, DNA size marker (ordinate values in bp).

L. We carried out stool analysis for Mrs. Xiao, and eggs were also found. However, the symptoms of Mrs. Xiao were relieved. No egg was found in the younger sister, husband, and daughter. Mrs. Xiao continued treatment.

This study was approved by the Medical Ethics Review Committee of National Institute of Parasitic Diseases, Chinese Center for Disease Control and Prevention. Meanwhile, the Medical Ethics Review Committee of National Institute of Parasitic Diseases, Chinese Center for Disease Control and Prevention exempted from individual informed consent targeting parasitological, serum antibody, and molecular identification of eggs from the patients' stool samples.

\section{DISCUSSION}

In this report, 4 members of a family were infected with fascioliasis after ingesting freshwater cultivated plants (water dropwort) contaminated with Fasciola metacercariae. Four clinical periods in fascioliasis can be distinguished [3]. The incubation period spans from consuming metacercariae to symptom appearance; in the acute/invasive period, fluke migration up to the bile ducts occurs; the latent stage includes maturation and oviposition; the chronic/obstructive period may develop after months to years of infection. Patients are usually diagnosed between the 2nd and 4th periods. Symptoms of eosinophilia, fever, RUQ abdominal pain without jaundice, hypodense liver lesions on $\mathrm{CT}$, and exposure history (history of consuming freshwater vegetables that may be improperly washed) suggests acute fascioliasis.

However, a negative history does not rule out fascioliasis. In the acute period, high fever, elevated ESR, anemia, leukocytosis, and high eosinophilia (may reach $70 \%$ ) are common in infected individuals [24]. Mrs. Xiao had the above described symptoms; the clinical presentation and liver damage probably pointed to the unique diagnosis. Aminotransferase levels are usually normal or slightly increased, while bilirubin levels are typically in the normal range, as found in Mrs. Xiao. However, a report described about 28 patients with acute fascioliasis who predominantly had epigastric pain, fatigue, fever, and RUQ abdominal pain, with elevated eosinophilia, ALT levels and acute-phase protein amounts in laboratory findings [25]. Moreover, eosinophilia increased strikingly in this disease [64]. According to Pavlović et al. [27], high ALP levels, as well as significantly elevated eosinophilia and low hemoglobin, are the most significant laboratory features of human fascioliasis, as was in the main case presented here. However, Valero et al. [8] found that $\gamma$-glutamyl transferase (GGT) provides information on penetration of young flukes into the bile ducts of sheep. GGT is the best marker, while AST is less sensitive [28].

The diagnosis of fascioliasis is complex and requires application of both direct and indirect diagnostic methods. Diagnosis of Fasciola infection traditionally relies on detecting eggs in stool samples or bile specimens. The Kato-katz method is commonly used for egg detection in fecal specimens. However, this approach is extremely limited in early stages of fascioliasis [29]. Moreover, the low sensitivity of this method is due to the low amount of feces included in a slide. It is difficult to find eggs, which leads to misdiagnosis ultimately. Therefore, nega- 
tive stool results do not rule out fascioliasis. Indeed, Fasciola spp. needs at least 3 to 4 months to develop to sexual maturity in humans [30].

Specific antibodies to Fasciola are detectable within 2 to 4 weeks after infection, i.e., 5 to 7 weeks before eggs appear in stool. Early diagnosis of this disease can be performed by serum assessment [24]. However, for a rare parasitic infection, such as fascioliasis, there is a lack of registered diagnostic tests in most areas of China. Moreover, serological detection always showing cross-reaction with other helminths, this method was not suitable for community surveys in rural endemic areas, especially in developing countries [30]. Therefore, the diagnosis and treatment are often problematic and delayed in most cases.

Migration of juvenile Fasciola through the liver in the invasive stage could cause hepatic lesions. Pathological sections show micro-abscesses and tunnel-like areas of parenchymal necrosis [31]. Imaging should be applied as auxiliary diagnosis, which may show abscesses and tunnels caused by the migrating young flukes or those in the biliary passage (chronic infection) [32]. The abdominal MRI finding in the current patient showing hypodense clustered lesions on the periphery of both hepatic lobes was compatible with the hepatic phase of fascioliasis. However, images seen on ultrasound and CT may sometimes be confused with malignancy or stones. The most useful diagnostic test for detecting the bile ducts is cholangiography by endoscopic retrograde cholangiopancreatography (ERCP). ERCP is quite important in evaluating symptomatic patients, especially in those with cholestatic syndrome, colicky pain, and abnormal liver function, or those in whom the parasitic disease has caused systemic involvement [33].

The first-line treatment of human fascioliasis consists of a single oral dose $(10 \mathrm{mg} / \mathrm{kg})$ of triclabendazole, which is highly effective against mature and immature flukes, safe, and easy to use [34]. Treatment should be repeated when a single dose fails to cure the infection [26].

In conclusion, human fascioliasis is indeed neglected in China. This zoonotic parasitic disease is very difficult to diagnose, because it sometimes appears with atypical and severe clinical presentations. Problematic and delayed diagnosis is especially risky in non-endemic areas, where clinicians are not familiar with this disease. The present report provides detailed clinical and detection information, and could inspire new diagnostic approaches in mitigating parasite transmission and virulence in humans.

\section{ACKNOWLEDGMENTS}

The authors are grateful to the patients for enthusiastic help in the collection of disease details regarding symptoms and samples for test. The project was funded by grants from the 4th Round of 3-Year Public Health Action Plan of Shanghai, China (no. 15GWZK0101), the Parasitic and Tropical Diseases Resource Center Project of National Science and Technology Basic Conditions Platform Program, the General Program Shanghai Municipal Commission of Health and Family Planning of China (no. 201640278), the Program for National Key Research and Development Program of China (grant nos. 2016YFC1202000, 2016YFC1202005), National S and T Major Program (grant no. 2012ZX10004-220), and the Chinese Special Program for Scientific Research of Public Health (grant nos. 201502021, 201202019).

\section{CONFLICT OF INTEREST}

We have no conflict of interest related to this study.

\section{REFERENCES}

1. Mas-Coma S, Bargues MD, Valero MA. Fascioliasis and other plant-borne trematode zoonoses. Int J Parasitol 2005; 35: 12551278 .

2. Mas-Coma S, Valero MA, Bargues MD. Fascioliasis. Adv Exp Med Biol 2014; 766: 77-114.

3. Mas-Coma S, Valero MA, Bargues MD. Chapter 2. Fasciola, lymnaeids and human fascioliasis, with a global overview on disease transmission, epidemiology, evolutionary genetics, molecular epidemiology and control. Adv Parasitol 2009; 69: 41-146.

4. Kim AJ, Choi CH, Choi SK, Shin YW, Park YK, Kim L, Choi SJ, Han JY, Kim JM, Chu YC, Park IS. Ectopic human Fasciola hepatica infection by an adult worm in the mesocolon. Korean J Parasitol 2015; 53: 725-730.

5. Qureshi AW, Tanveer A, Mas-Coma S. Epidemiological analysis of human fascioliasis in northeastern Punjab, Pakistan. Acta Trop 2016; 156: 157-164.

6. Fürst T, Keiser J, Utzinger J. Global burden of human food-borne trematodiasis: a systematic review and meta-analysis. Lancet Infect Dis 2012; 12: 210-221.

7. Mas-Coma S, Agramunt VH, Valero MA. Neurological and ocular fascioliasis in humans. Adv Parasitol 2014; 84: 27-149.

8. Valero MA, Bargues MD, Khoubbane M, Artigas P, Quesada C, Berinde L, Ubeira FM, Mezo MM, Hernandez JL, Agramunt VH, Mas-Coma S. Higher physiopathogenicity by Fasciola gigantica than by the genetically close F. hepatica: experimental long-term follow-up of biochemical markers. Trans R Soc Trop Med Hyg 
2016; 110: 55-66.

9. Dalton JP, Robinson MW, Mulcahy G, O'Neill SM, Donnelly S. Immunomodulatory molecules of Fasciola hepatica: candidates for both vaccine and immunotherapeutic development. Vet Parasitol 2013; 195: 272-285.

10. Gironès N, Valero MA, García-Bodelón MA, Chico-Calero I, Punzón C, Fresno M, Mas-Coma S. Immune suppression in advanced chronic fascioliasis: an experimental study in a rat model. J Infect Dis 2007; 195: 1504-1512.

11. World Health Organization. Fact sheet on fascioliasis. In Action Against Worms. World Health Organization, Geneva, Switzerland. Newsletter 2008; 10: 1-8.

12. Gil LC, Díaz A, Rueda C, Martínez C, Castillo D, Apt W. Resistant human fasciolasis: report of four patients. Rev Med Chil 2014; 142: 1330-1333 (in Spanish).

13. Chen JZ, Xu F, Wu RZ, Guo RN, Xing DZ, Huang DL, Li SW, Lin SX, Huang JM, Liu YR, Chen DY. Human infections with Fasciola hepatica and Fasciola gigantica in Hainan province. Chin J Parasitol Parasit Dis 1994; 12: 243 (in Chinese).

14. Fan D, Li P, Sun H, Wang ZH, She B. CT features of liver abscesses caused by the Fasciola hepatica infection. Chin J Radiol 2006; 40: 191-194 (in Chinese).

15. Huang ZM, Xing LY, Fan B, Zhao W, Fan ZH. Report on a patient infected with Fasciola hepatica. Chin Trop Med 2006; 16: 1624 (in Chinese).

16. Huang ZM, Xing LY, Li LJ, Fan B. Two cases of fascioliasis hepatica. Parasit Infect Dis 2009; 7: 172 (in Chinese).

17. Chen JX, Chen MX, Ai L, Xu XN, Jiao JM, Zhu TJ, Su HY, Zang W, Luo JJ, Guo YH, Lv S, Zhou XN. An Outbreak of human fascioliasis gigantica in Southwest China. PLoS One 2013; 8: e71520.

18. Chen MX, Chen SH, Hu W, Li J, Ai L, Chen JX. Parasitological and molecular detection on a young man of human fasciolosis diagnosed in Guizhou, China. Trop Biomed 2017 (in press).

19. Valero MA, Periago MV, Pérez-Crespo I, Rodríguez E, Perteguer MJ, Gárate T, González-Barberá EM, Mas-Coma S. Assessing the validity of an ELISA test for the serological diagnosis of human fascioliasis in different epidemiological situations. Trop Med Int Health 2012; 17: 630-636.

20. Suhardono, Roberts JA, Copeman DB. Variations in the survival of Fasciola gigantica eggs in bovine dung stored in the sun as opposed to the shade. Trop Anim Health Prod 2006; 38: 379-382.

21. Ai L, Dong SJ, Zhang WY, Elsheikha HM, Mahmmod YS, Lin RQ, Yuan ZG, Shi YL, Huang WY, Zhu XQ. Specific PCR-based assays for the identification of Fasciola species: their develop- ment, evaluation and potential usefulness in prevalence surveys. Ann Trop Med Parasitol 2010; 104: 65-72.

22. Luton $\mathrm{K}$, Walker D, Blair D. Comparisons of ribosomal internal transcribed spacers from two congeneric species of flukes (Platyhelminthes: Trematoda: Digenea). Mol Biochem Parasitol 1992; 56: 323-327.

23. Bowles J, Blair D, McManus DP. Genetic variants within the genus Echinococcus identified by mitochondrial DNA sequencing. Mol Biochem Parasitol 1992; 54: 165-173.

24. Soliman MF. Epidemiological review of human and animal fascioliosis in Egypt. J Infect Dev Ctries 2008; 2: 182-189.

25. Saba R, Korkmaz M, Inan D, Mamikoglu L, Turhan O, Gunseren F, Cevikol C, Kabaalioğlu A. Human fascioliosis. Clin Microbiol Infect 2004; 10: 385-387.

26. Dauchy FA, Vincendeau P, Lifermann F. Eight cases of fascioliosis: clinical and microbiological features. Med Mal Infect 2006; 36: 42-46.

27. Pavlović M, Dakić Z, Milosević B, Korać M, Brmbolić B, Dzamić A. Human case of fasciolosis in Serbia treated with triclabendazole. Vojnosanit Pregl 2014; 71: 202-206.

28. Kozat S, Denizhan V. Glucose, lipid, and lipoprotein levels in sheep naturally infected with Fasciola hepatica. J Parasitol 2010; 96: 657-659.

29. Mas-Coma S, Bargues MD, Valero MA. Diagnosis of human fascioliasis by stool and blood techniques: update for the present global scenario. Parasitology 2014; 141: 1918-1946.

30. Espino AM, Díaz A, Pérez A, Finlay CM. Dynamics of antigenemia and coproantigens during a human Fasciola hepatica outbreak. J Clin Microbiol 1998; 36: 2723-2726.

31. Pagola Serrano MA, Vega A, Ortega E, Gonzales A. Computed tomography of hepatic fascioliosis. J Comput Assist Tomog 1987; 11: 269-272.

32. Patel NU, Bang TJ, Dodd GD 3rd. CT findings of human Fasciola hepatica infection: case reports and review of the literature. Clin Imaging 2016; 40: 251-255.

33. Roig GV. Hepatic fascioliasis in the Americas: a new challenge for therapeutic endoscopy. Gastrointest Endosc 2002; 56: 315317.

34. Marcos LA, Tagle M, Terashima A, Bussalleu A, Ramirez C, Carrasco C, Valdez L, Huerta-Mercado J, Freedman DO, Vinetz JM, Gotuzzo E. Natural history, clinicoradiologic correlates, and response to triclabendazole in acute massive fasciolosis. Am J Trop Med Hyg 2008; 78: 222-227. 